hep-ph/9905405

FTUV/99-21

$\mathrm{IFIC} / 99-22$

SISSA 31/99/EP

December 15, 1999

\title{
A potential test of the CP properties and Majorana nature of neutrinos
}

\author{
S. Pastor ${ }^{a}$ " J. Segura ${ }^{b}$, V.B. Semikoz ${ }^{a}$ and J.W.F. Valle ${ }^{d}$ \\ ${ }^{a}$ SISSA-ISAS and INFN, Sezione di Trieste \\ Via Beirut 2-4,I-34013 Trieste, ITALY \\ ${ }^{b}$ Instituto de Bioingeniería \& \\ Departamento de Estadística y Matemática Aplicada \\ Universidad Miguel Hernández, Edificio La Galia \\ 03206 Elche, Alicante, SPAIN \\ ${ }^{c}$ Institute of Terrestrial Magnetism, the Ionosphere and Radio \\ Wave Propagation of the Russian Academy of Sciences, IZMIRAN \\ Troitsk, Moscow region, 142092, RUSSIA \\ ${ }^{d}$ Instituto de Física Corpuscular - C.S.I.C. \\ Departament de Física Teòrica, Universitat de València \\ 46100 Burjassot, València, SPAIN \\ http://neutrinos.uv.es
}

\begin{abstract}
The scattering of solar neutrinos on electrons may reveal their CP properties, which are particularly sensitive to their Majorana nature. The cross section is sensitive to the neutrino dipole moments through an interference of electro-magnetic and weak amplitudes. We show how future solar neutrino experiments with good angular resolution and low energy threshold, such as Hellaz, can be sensitive to the resulting azimuthal asymmetries in event number, and could therefore provide valuable information on the $\mathrm{CP}$ properties and the nature of the neutrinos, provided the solar magnetic field direction is fixed.
\end{abstract}

\footnotetext{
1 E-mail: pastor@sissa.it

2 E-mail: javi.segura@umh.es

3 E-mail: semikoz@izmiran.rssi.ru

4 E-mail: valle@flamenco.ific.uv.es
} 


\section{Introduction}

It has long been realized that, on general grounds, gauge theories generally predict that, if neutrinos are massive, they should be Majorana particles, unless protecting symmetries are imposed or arise accidentally [1]. Even though lepton-number-violating processes such as neutrinoless double beta decay are intrinsically related to the Majorana nature of neutrinos in a gauge theory [2], their search as so far yielded only negative results [3]. It has also been shown in the early 80's that gauge theories with Majorana neutrinos contain additional CP violating phases without analogue in the quark sector [1]. Although these are genuine physical parameters of the theory, as they show up in $\Delta L=2$ neutrino oscillations [4], their effects are also suppressed by the smallness of neutrino masses, relative to the typical neutrino energies available at accelerator and even reactor experiments.

Among the non-standard properties of neutrinos the electro-magnetic dipole moments [5, 6, 7, 8, 9] play an important conceptual role, since they can potentially signal the Majorana nature of neutrinos. Neutrino transition electro-magnetic moments [5] are especially interesting because their effects can be resonantly enhanced in matter [10 and provide an attractive solution of the solar neutrino problem [11] without running in conflict with astrophysics [12].

For pure left-handed neutrinos the weak interaction amplitude on electrons does not interfere with that of the electro-magnetic interaction, since the weak interaction preserves neutrino helicity while the electro-magnetic does not. As a result the cross section depends quadratically on the neutrino electro-magnetic form factors. However if there exists a process capable of converting part of the initially fully polarized neutrinos, then an interference term arises proportional to the neutrino electro-magnetic form factors, as pointed out e.g. in ref. [13]. This term depends on the angle between the component of the neutrino spin transverse to its momentum and the momentum of the outgoing recoil electron. Therefore the number of events measured in an experiment exhibits an asymmetry with respect to the above defined angle. The asymmetry will not show up in terrestrial experiments even with stronger magnetic fields, since only in the Sun the neutrino depolarization would be resonant and only in the solar convective zone one will find a magnetic field extended over such a region (about a third of the solar radius wide). At earth-bound laboratory experiments the helicity-flip could be caused only by the presence of a neutrino mass and is therefore small [14], in a way analogous to the case

of neutrino $\Delta L=2$ lepton-number-violating neutrino oscillations [4]. Exotic couplings to 
scalars might change this feature, but there are relatively strong limits. In contrast for a relatively modest large-scale solar magnetic field in the convective region $B_{\perp} \sim 10^{4} \mathrm{G}$ and a neutrino magnetic moment of the order $10^{-11} \mu_{B}$, where $\mu_{B}$ is the Bohr magneton, one has $\mu_{\nu} B_{\perp} L \sim 1$ since $L \sim L_{\text {conv }} \simeq 2 \times 10^{10} \mathrm{~cm}$ is the width of the convective zone. Such a spin-flip process may depolarize the solar neutrino flux at a level where neutrino electro-magnetic properties may reveal the Majorana nature of neutrinos (or alternatively, the solar magnetic field structure).

In this paper we show that the resonant enhancement of neutrino conversions induced by Majorana transition moments can provide valuable hints on the true nature of neutrinos and their CP properties, in a way which is not suppressed by the small neutrino mass. Our proposed test requires the careful investigation of neutrino-electron scattering for neutrinos from the Sun at future solar neutrino experiments with good angular resolution and low energy threshold. One such proposed experiment is Hellaz [15]. For completeness and pedagogy we also include a discussion of the Dirac-type magnetic moment or electric dipole moment [16].

\section{Neutrino Electro-Magnetic Properties}

The most general effective interaction Lagrangian describing the electro-magnetic properties of Majorana neutrinos has been first given in ref. [5] in terms of the fundamental two-component spinors. The connection with conventional four-component description can be found in ref. [1]. Other equivalent presentations are given in [8, 9] and the corresponding matrix element between one-particle neutrino states for a real $\left(q^{2}=0\right)$ photon, can be written as 12

$$
<p^{\prime}, s^{\prime}, j\left|\mathcal{L}_{e f f}\right| p, s, i>=\bar{u}_{j}^{s^{\prime}}\left(p^{\prime}\right) \Gamma_{\lambda}^{i j}\left(p, p^{\prime}\right) u_{i}^{s}(p) A^{\lambda}(q)=\bar{u}_{j}^{s^{\prime}}\left(p^{\prime}\right) i \sigma_{\lambda \rho} q^{\rho}\left(\mu_{i j}+i d_{i j} \gamma_{5}\right) u_{i}^{s}(p) A^{\lambda}(q)
$$

where $i, j$ denote the mass labels of the neutrinos, the indices $s$ and $s^{\prime}$ specify helicities, while the $u$ 's are the standard wave functions of the Dirac equation and $q=p-p^{\prime}$. Here $\mu_{i j}$ and $d_{i j}$ are the magnetic and electric dipole moments, respectively. From the hermiticity condition for the Lagrangian one can relate [6, 7] the form factors of the $i \rightarrow j$ process and its inverse,

$$
\mu_{i j}=\mu_{j i}^{*} \quad d_{i j}=d_{j i}^{*}
$$


Note that in the diagonal $i=j$ case, both $\mu$ and $d$ must be real according to eq. (21). For further constraints on the form factors $\mu_{i j}$ and $d_{i j}$ one must assume something about the neutrino nature and/or invariance under the CP symmetry [5, 8]. These interactions arise only from loops in a gauge theory like the Standard Model and are therefore calculable from first principles. However, in most gauge theories magnetic moments are expected to be small. For discussions see ref. [8].

Majorana neutrinos can have only off-diagonal $(i \neq j)$ form factors, called transition moments [5], while if the neutrinos are Dirac particles, just as the charged leptons, both diagonal and off-diagonal moments can exist. Let us assume that the effective Lagrangian is invariant under a CP transformation, $\mathcal{L}_{\text {eff }}=C P \mathcal{L}_{\text {eff }}(C P)^{-1}$. A Dirac field transforms under CP as $C P \Psi_{i}(\vec{x}, t)(C P)^{-1}=\eta_{i} C \Psi_{i}^{*}(-\vec{x}, t)$, where $\eta_{i}$ is a phase factor and $C$ is the charge conjugation matrix $\left(C^{-1}=C^{\dagger}=C^{T}=-C\right)$ [7, 8, 9]. If we apply this to the $\sigma_{\mu \nu}$-part of the effective Lagrangian, the CP-transformed $i \rightarrow j$ part will contribute to the $j \rightarrow i$ process and vice versa. The result for $\Gamma_{\lambda}$ implies that

$$
\bar{u}_{j}\left(p^{\prime}\right) \Gamma_{\lambda}^{i j}(q) u_{i}(p)=\eta_{i}^{*} \eta_{j} \bar{u}_{j}\left(p^{\prime}\right) \bar{\Gamma}_{\lambda}^{i j}(q) u_{i}(p)
$$

where $\bar{\Gamma}_{\lambda}^{i j}$ is equal to $\Gamma_{\lambda}^{i j}$ with the change $\gamma_{5} \rightarrow-\gamma_{5}$. Eq. (3) implies that the form factors obey the relations

$$
\frac{\mu_{i j}^{*}}{\mu_{i j}}=-\frac{d_{i j}^{*}}{d_{i j}}=\eta_{i} \eta_{j}^{*}
$$

A Majorana neutrino is its own anti-particle. It is easy to check in this case that in eq. (目) both the $i j$ and the $j i$ terms in the Lagrangian will contribute to the $i j$ form factors. One finds that for mass eigenstates [7]

$$
<p^{\prime}, j\left|\mathcal{L}_{e f f}\right| p, i>=\bar{u}_{j}\left(p^{\prime}\right) i \sigma_{\lambda \rho} q^{\rho}\left[\left(\mu_{i j}-\mu_{j i}\right)+i\left(d_{i j}-d_{j i}\right) \gamma_{5}\right] u_{i}(p) A^{\lambda}
$$

Finally, from the hermiticity condition eq. (2) one gets $\mu_{i j}-\mu_{j i}=2 i \operatorname{Im}\left(\mu_{i j}\right)$ and $d_{i j}-d_{j i}=$ $2 i \operatorname{Im}\left(d_{i j}\right)$. Therefore we conclude that a Majorana neutrino has no diagonal electromagnetic factors and that the transition form factors $\mu_{i j}$ and $d_{i j}$ are both pure imaginary, irrespective of whether or not one assumes CP invariance [5]. Thus Majorana neutrinos can only possess transition magnetic or electric dipole moments. Let us now check whether $\mathrm{CP}$ invariance restricts them. If $\mathrm{CP}$ is conserved, a Majorana neutrino is a CP eigenstate, with a phase $\eta_{C P}= \pm i$ [5]. Considering the invariance of $\mathcal{L}$ for the Majorana case under $\mathrm{CP}$ one gets a condition similar to eq. (3) and eq. (团). There are two physically interesting cases to consider: two neutrino species involved in eq. (1) can be either both active, weakly interacting neutrinos, or one of them can be sterile. Moreover, for each of these cases, 


\begin{tabular}{|c|c|c|}
\hline Case & Hermiticity & Hermiticity + CP \\
\hline Dirac $i=j$ & $\mu_{i i}$ and $d_{i i}$ real & $d_{i i}=0$ \\
\hline Dirac $i \neq j$ & $\mu_{i j}=\mu_{j i}^{*}$ and $d_{i j}=d_{j i}^{*}$ & $\mu_{i j}$ and $i d_{i j}$ relatively real \\
\hline Majorana $i=j$ & $\mu_{i i}=d_{i i}=0$ & - \\
\hline \multirow{2}{*}{ Majorana $i \neq j$} & $\mu_{i j}$ and $d_{i j}$ & Case $(+-): d_{i j}=0$ \\
\cline { 3 - 3 } & pure imaginary & Case $(++): \mu_{i j}=0$ \\
\hline
\end{tabular}

Table 1: General properties of neutrino electro-magnetic dipole moments

there are two possible $\mathrm{CP}$-conserving cases, depending on the relative $\mathrm{CP}$ sign of the neutrinos involved

1. Case $(+-):\left(\eta_{i}, \eta_{j}\right)=( \pm i, \mp i)$, then $\mu_{i j}$ survives and $d_{i j}=0$. This is a pure magnetic transition, and includes the Dirac-type magnetic moment if one of the neutrinos is sterile.

2. Case $(++):\left(\eta_{i}, \eta_{j}\right)=( \pm i, \pm i)$, then $\mu_{i j}=0$ and $d_{i j}$ survives [0]. This is a pure electric transition.

On the contrary, as emphasized by Wolfenstein [5], if $\mathrm{CP}$ is not conserved both magnetic and electric dipole moments will contribute to the neutrino-electron scattering cross section. The general properties of Dirac and Majorana neutrino electro-magnetic dipole moments are summarized in table 1 .

Note that the above discussion is completely general and covers all types of Majorana transition moments, active-active and active-sterile. In particular it covers the activesterile case with zero mass splitting (Dirac diagonal case). In what follows we will focus mainly on active-active Majorana transition moments, as well as the Dirac diagonal case.

\section{Dipole moments for flavor states}

We have discussed so far the restrictions upon the neutrino electro-magnetic dipole moments for mass eigenstates. Since we are interested in possible interference terms between 
weak and electro-magnetic interactions in neutrino-electron scattering, we shall present the corresponding matrix elements in terms of flavor states. For simplicity we will restrict ourselves to the two-generation case, and for definiteness the $\nu_{e}-\nu_{a}$ pair, where $\nu_{a}$ can be an active neutrino (for instance $\nu_{\mu}$ ) or a sterile neutrino $\nu_{s}$. In this case the mixing matrix contains a CP violating phase for the case of Majorana neutrinos [1, 4. Such phase is absent if the two neutrinos are Dirac type, since in this case it can be removed by field redefinition, as expected in analogy with the quark sector, where CP violation sets in only for three generations.

The $\bar{u} \Gamma_{\lambda} u$ matrix element can be written as in eq. (11) but for flavor eigenstates $\nu_{e, a}$, with $\mu_{\nu_{e} \nu_{a}}$ and $d_{\nu_{e} \nu_{a}}$. Here the restrictions on $\mu$ and $d$ for Dirac mass states still apply, and in particular for the diagonal case both $\mu_{\nu_{e}}$ and $d_{\nu_{e}}$ are real.

The CP-violating phase which is present in the mixing matrix of a theory with two Majorana neutrinos may be introduced as the $e^{i \beta}$ phase in the $2 \times 2$ mixing matrix,

$$
\left(\begin{array}{c}
\nu_{e} \\
\nu_{a}
\end{array}\right)=\left(\begin{array}{cc}
c & s e^{i \beta} \\
-s & c e^{i \beta}
\end{array}\right)\left(\begin{array}{c}
\nu_{1} \\
\nu_{2}
\end{array}\right)=\left(\begin{array}{cc}
c & s \\
-s & c
\end{array}\right)\left(\begin{array}{cc}
1 & 0 \\
0 & e^{i \beta}
\end{array}\right)\left(\begin{array}{l}
\nu_{1} \\
\nu_{2}
\end{array}\right)
$$

Here $c \equiv \cos \theta, s \equiv \sin \theta$, where $\theta$ denotes the leptonic mixing angle. Let us define now in eq. (5) the real parameters $\mu_{i j}^{\prime} \equiv \operatorname{Im}\left(\mu_{i j}\right)$ and $d_{i j}^{\prime} \equiv \operatorname{Im}\left(d_{i j}\right)$. The expression for eq. (5) is then as follows

$$
\bar{u}_{j}\left(p^{\prime}\right) i \sigma_{\lambda \rho} q^{\rho} 2 i\left(\mu_{i j}^{\prime}+i d_{i j}^{\prime} \gamma_{5}\right) u_{i}(p)
$$

If we introduce now the weak states according to eq. (6), one gets two contributions to the $\nu_{e}-\nu_{a}$ amplitude, corresponding to $i=1, j=2$ and $i=2, j=1$. Using the hermiticity condition one has $\mu_{21}^{\prime}=-\mu_{12}^{\prime}$ and $d_{21}^{\prime}=-d_{12}^{\prime}$, and one can define the electro-magnetic dipole moments for flavor states as follows $\left(\kappa=\mu, d\right.$ and note that $\left.\kappa_{a e}=\kappa_{e a}^{*}\right)$

$$
\begin{gathered}
\kappa_{e a} \equiv \kappa_{\nu_{e} \nu_{a}}=2 i\left(c^{2} e^{i \beta}+s^{2} e^{-i \beta}\right) \kappa_{12}^{\prime}=2 i\left(\cos \beta+i\left(c^{2}-s^{2}\right) \sin \beta\right) \kappa_{12}^{\prime} \\
\kappa_{a e} \equiv \kappa_{\nu_{a} \nu_{e}}=2 i\left(c^{2} e^{-i \beta}+s^{2} e^{i \beta}\right) \kappa_{21}^{\prime}=2 i\left(\cos \beta-i\left(c^{2}-s^{2}\right) \sin \beta\right) \kappa_{21}^{\prime}
\end{gathered}
$$

We conclude then that a pair of Majorana neutrinos (weak states) has, in general, complex dipole moments. This is a consequence of the $\mathrm{CP}$ phase from the mixing matrix. The particular CP-conserving cases correspond to the values $\beta=0, \pi / 2$. Therefore, when assuming CP invariance the electro-magnetic current for neutrinos takes the forms

$$
\begin{aligned}
& \beta=\pi / 2 \quad \Longrightarrow \quad \bar{u}_{\nu_{a}}\left(p^{\prime}\right) i \sigma_{\lambda \rho} q^{\rho} \operatorname{Re}\left(\mu_{e a}\right) u_{\nu_{e}}(p)+\text { h.c. } \\
& \beta=0 \quad \Longrightarrow \quad-\bar{u}_{\nu_{a}}\left(p^{\prime}\right) i \sigma_{\lambda \rho} q^{\rho} \operatorname{Im}\left(d_{e a}\right) \gamma_{5} u_{\nu_{e}}(p)+\text { h.c. }
\end{aligned}
$$

The first case is the limit that we considered in our previous paper [17]. 


\section{Neutrino-electron scattering cross sections}

We consider the scattering of neutrinos on electrons when the initial flux of neutrinos is not completely polarized, i.e. there exists a mechanism that converts part of the initial left-handed electron neutrinos (produced in weak processes) into right-handed ones. We assume that this is a consequence of the presence of non-zero neutrino electro-magnetic dipole moments. The Sun seems to be the only physical situation where such depolarization process can occur.

Let us consider the scattering $\nu\left(k_{1}\right)+e^{-}\left(p_{1}\right) \rightarrow \nu\left(k_{2}\right)+e^{-}\left(p_{2}\right)$, in the coordinate frame where the initial electron is at rest. The four-vectors of the particles involved, taking into account conservation of momenta, are the following

$$
k_{1}=\left(\omega, \vec{k}_{1}\right) \quad p_{1}=\left(m_{e}, \overrightarrow{0}\right) \quad k_{2}=\left(\omega-T, \vec{k}_{2}\right) \quad p_{2}=\left(m_{e}+T, \vec{p}_{2}\right)
$$

where $T$ is the electron recoil energy and $p_{1}^{2}=p_{2}^{2}=m_{e}^{2}$. From now on we consider the limit of ultra-relativistic neutrinos, i.e. $k_{1}^{2}=k_{2}^{2} \simeq 0$ and the low-energy limit $\left(\omega \ll M_{W}\right)$. There are two contributions to the scattering process: weak and electro-magnetic. As in the previous section we will consider the case of two neutrino species. There are two inequivalent physical situations, namely (i) $\nu_{e}-\nu_{\mu}$ and (ii) $\nu_{e}-\nu_{s}$, where $\nu_{s}$ is a sterile type neutrino.

Following the conventions of ref. [18 for $f=\nu_{e}, \nu_{\mu}$, the corresponding weak matrix amplitudes for Dirac and Majorana neutrinos are, respectively

$$
\begin{gathered}
M_{W_{f}}^{D}=-i 2 \sqrt{2} G_{F} \bar{u}_{f}^{r^{\prime}}\left(k_{2}\right) \gamma^{\mu} \frac{1-\gamma_{5}}{2} u_{f}^{r}\left(k_{1}\right) \bar{u}_{e}^{s^{\prime}}\left(p_{2}\right) \gamma_{\mu}\left(g_{f L} \frac{1-\gamma_{5}}{2}+g_{R} \frac{1+\gamma_{5}}{2}\right) u_{e}^{s}\left(p_{1}\right) \\
M_{W_{f}}^{M}=i 2 \sqrt{2} G_{F} \bar{u}_{f}^{r^{\prime}}\left(k_{2}\right) \gamma^{\mu} \gamma_{5} u_{f}^{r}\left(k_{1}\right) \bar{u}_{e}^{s^{\prime}}\left(p_{2}\right) \gamma_{\mu}\left(g_{f L} \frac{1-\gamma_{5}}{2}+g_{R} \frac{1+\gamma_{5}}{2}\right) u_{e}^{s}\left(p_{1}\right)
\end{gathered}
$$

and obviously zero for sterile neutrinos. Here $g_{e L}=\sin ^{2} \theta_{W}+1 / 2, g_{\mu L}=\sin ^{2} \theta_{W}-1 / 2$ and $g_{R}=\sin ^{2} \theta_{W}$. The electro-magnetic amplitudes are

$$
M_{a b}^{e m}=\frac{e}{q^{2}} \bar{u}_{b}^{r^{\prime}}\left(k_{2}\right) \sigma_{\lambda \rho} q^{\rho}\left(\mu_{a b}+i d_{a b} \gamma_{5}\right) u_{a}^{r}\left(k_{1}\right) \bar{u}_{e}^{s^{\prime}}\left(p_{2}\right) \gamma^{\lambda} u_{e}^{s}\left(p_{1}\right)
$$

where $a b$ denotes $\nu_{e} \nu_{\mu}$ or $\nu_{e} \nu_{s}$, and the form factors $\mu_{a b}$ and $d_{a b}$ depend on whether neutrinos are Dirac or Majorana.

In order to simplify the notation let us set $\mu_{a} \equiv \mu_{a a}$ and $d_{a} \equiv d_{a a}$ (both real) for the diagonal case, and $\mu \equiv \mu_{e a}=\mu_{a e}^{*}$ and $d \equiv d_{e a}=d_{a e}^{*}$ for the transition dipole moments, which are complex in general. We will perform the calculation of the differential cross 


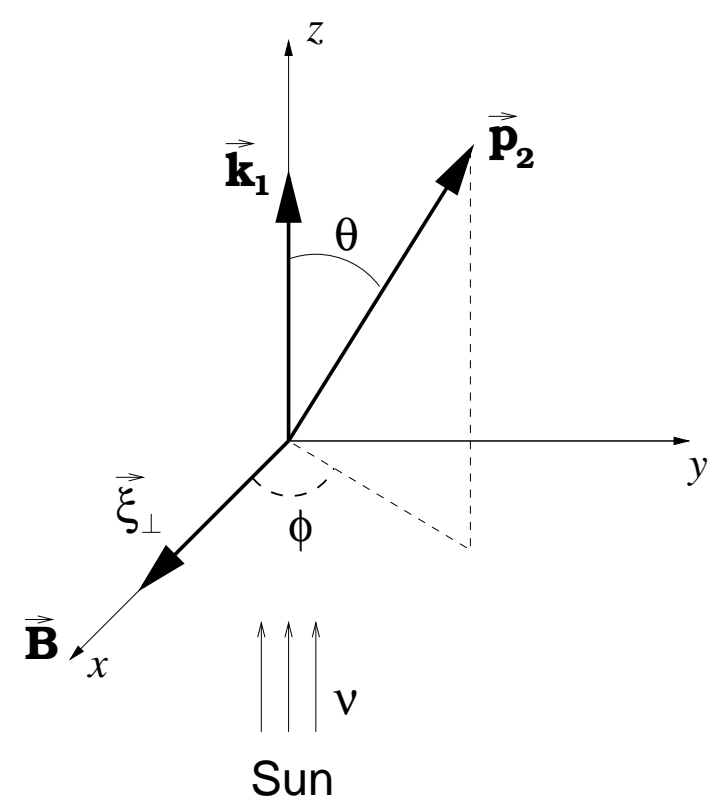

Figure 1: Coordinate system conventions.

section for neutrino-electron scattering without assuming CP invariance and in the two physical situations. It can be written as a sum of three terms,

$$
\frac{d \sigma}{d T d \phi}=\left(\frac{d \sigma}{d T d \phi}\right)_{w e a k}+\left(\frac{d \sigma}{d T d \phi}\right)_{e m}+\left(\frac{d \sigma}{d T d \phi}\right)_{i n t}
$$

that correspond to the purely weak, the purely electro-magnetic and the interference term, respectively. In the last equation $\phi$ is the azimuthal angle, as defined in figure 1 .

\subsection{Active-active case}

In this case $\left(\nu_{e}-\nu_{\mu}\right)$ the purely weak term can be written in a general form as

$$
\left(\frac{d \sigma}{d \phi d T}\right)_{\text {weak }}=\frac{G_{F}^{2} m_{e}}{\pi^{2}}\left[P_{e} h\left(g_{e L}, g_{R}\right)+P_{\bar{e}} h\left(g_{R}, g_{e L}\right)+P_{\mu} h\left(g_{\mu L}, g_{R}\right)+P_{\bar{\mu}} h\left(g_{R}, g_{\mu L}\right)\right]
$$

where we have defined $h(x, y) \equiv x^{2}+y^{2}(1-T / \omega)^{2}-x y m_{e} T / \omega^{2}$. Here $P_{A}\left(P_{\bar{A}}\right)$ is the probability of measuring $\nu_{A}=\nu_{e, \mu}\left(\bar{\nu}_{A}=\bar{\nu}_{e, \mu}\right)$, and from unitarity $P_{e}+P_{\bar{e}}+P_{\mu}+P_{\bar{\mu}}=1$. The purely electro-magnetic term in the presence of transition dipole moments is

$$
\left(\frac{d \sigma}{d T d \phi}\right)_{e m}=\frac{\alpha^{2}}{2 m_{e}^{2}}\left(\frac{1}{T}-\frac{1}{\omega}\right)\left[\frac{|\mu|^{2}+|d|^{2}}{\mu_{B}^{2}}+2\left(P_{\bar{e}}+P_{\mu}-P_{e}-P_{\bar{\mu}}\right) \frac{\operatorname{Im}\left(\mu d^{*}\right)}{\mu_{B}^{2}}\right]
$$


Note that in the limit of zero mixing only $\nu_{e}$ and $\bar{\nu}_{\mu}$ are present $\left(P_{\bar{e}}=0=P_{\mu}\right.$ and $P_{e}+P_{\bar{\mu}}=1$ ), so that the last equation reduces to the form (see e. g. [19])

$$
\left(\frac{d \sigma}{d T d \phi}\right)_{e m}=\frac{\alpha^{2}}{2 m_{e}^{2}}\left(\frac{1}{T}-\frac{1}{\omega}\right) \frac{|\mu-i d|^{2}}{\mu_{B}^{2}}
$$

It will be convenient in order to calculate the interference term of the cross section to describe the flux of initial neutrinos in terms of a density matrix $\rho$ which generalizes the usual to account for the case of two different flavors $A, B$. The neutrino part of the amplitude squared $M M^{\prime} \dagger$ is thus calculated as follows

$$
\sum_{\text {spins }}\left[\bar{u}_{B}\left(k_{2}\right) M u_{A}\left(k_{1}\right)\right]\left[\bar{u}_{A}\left(k_{2}\right) M^{\prime} u_{B}\left(k_{1}\right)\right]^{\dagger}=\operatorname{Tr}\left[M \rho_{A B}\left(k_{1}\right) \bar{M}^{\prime} \dagger \hat{k}_{2}\right]
$$

where $\bar{M}^{\prime \dagger}=\gamma_{0} M^{\prime \dagger} \gamma_{0}$ and $\hat{x} \equiv \gamma_{\lambda} x^{\lambda}$. The density matrix can be written as a function of the different neutrino probabilities and the components of the corresponding polarization vectors as follows

$$
\rho_{A B}\left(k_{1}\right)=\frac{1}{2}\left(P_{A}+P_{\bar{B}}+\left(P_{\bar{B}}-P_{A}\right) \gamma_{5}\right) \hat{k}_{1}+\frac{1}{4} \hat{\xi}_{\perp}^{\bar{A} B} \hat{k}_{1}\left(1+\gamma_{5}\right)+\frac{1}{4} \hat{k}_{1} \hat{\xi}_{\perp}^{A \bar{B}}\left(1-\gamma_{5}\right)
$$

where the polarization four-vectors $\xi_{\perp}^{\bar{A} B}=\left(0, \vec{\xi}_{\perp}^{\bar{A} B}\right)$ are orthogonal to the neutrino momentum, i.e. $k_{1} \cdot \xi_{\perp}^{\bar{A} B}=0=k_{1} \cdot \xi_{\perp}^{\bar{B} A}$ and $\left|\vec{\xi}_{\perp}^{\bar{A} B}\right|=2 \sqrt{P_{\bar{A}} P_{B}}$.

Note that in the case of one Dirac neutrino $A=B$ this reproduces the result given in the appendix $\mathrm{E}$ of ref. [20], namely

$$
\rho_{A}\left(k_{1}\right)=\frac{1}{2}\left(1-\xi_{\|} \gamma_{5}-\hat{\xi}_{\perp} \gamma_{5}\right) \hat{k}_{1}
$$

where $\vec{\xi}$ is the normalized polarization vector at the neutrino's rest frame, with $\left|\overrightarrow{\xi_{\perp}}\right|=$ $2 \sqrt{P_{A}\left(1-P_{A}\right)}$ and $\xi_{\|}=2 P_{A}-1$.

It is important to remark that the transversal component $\vec{\xi}_{\perp}$ of the neutrino polarization vectors are aligned along the direction of the solar magnetic field $\vec{B}_{\odot} 21$.

For ultra-relativistic neutrinos the interference term arises only if the initial flux contains some mixture of right-handed neutrinos. Note that in this subsection we restrict to the simple situation where the initial $\nu_{e}$ convert to $\bar{\nu}_{\mu}$ through a neutrino transition dipole moment (zero neutrino mixing). Then one sets $P_{\mu}=P_{\bar{e}}=0$, so that $\vec{\xi}_{\perp}^{\bar{e} \mu}=\overrightarrow{0}$. This is the process that can occur in the Sun. The expression for the interference term is found to be

$$
\begin{aligned}
\left(\frac{d \sigma}{d T d \phi}\right)_{i n t}=- & \frac{\alpha G_{F}}{4 \sqrt{2} \pi m_{e} T}\left[\left(\frac{\operatorname{Re}(\mu)+\operatorname{Im}(d)}{\mu_{B}}\right) \vec{p}_{2} \cdot \vec{A}_{M}(T, \omega)\right. \\
& \left.+\left(\frac{\operatorname{Re}(d)-\operatorname{Im}(\mu)}{\mu_{B}}\right) \hat{\vec{k}}_{1} \cdot\left(\vec{p}_{2} \times \vec{A}_{M}(T, \omega)\right)\right]
\end{aligned}
$$


where $\hat{\vec{k}}_{1} \equiv \vec{k}_{1} / \omega$ and we have defined

$$
\vec{A}_{M}(T, \omega) \equiv\left[\left(g_{e L}+g_{\mu L}+2 g_{R}\right)\left(2-\frac{T}{\omega}\right)+\left(g_{e L}-g_{\mu L}\right) \frac{T}{\omega}\right] \vec{\xi}_{\perp}^{e} \bar{\mu}
$$

Note that eq. (20) depends explicitly on the azimuthal angle $\phi$. Choosing the coordinate system as shown in figure 1 , this dependence is like $\cos \phi$ or $\sin \phi$, since it is easily checked that

$$
\begin{gathered}
\vec{p}_{2} \cdot \vec{\xi}_{\perp}=\left|\vec{p}_{2}\right| \sin \theta\left|\vec{\xi}_{\perp}\right| \cos \phi=\sqrt{2 m_{e} T\left(1-\frac{T}{T_{\text {max }}}\right)}\left|\vec{\xi}_{\perp}\right| \cos \phi \\
\hat{\vec{k}}_{1} \cdot\left(\vec{p}_{2} \times \vec{\xi}_{\perp}\right)=-\left|\vec{p}_{2}\right| \sin \theta\left|\vec{\xi}_{\perp}\right| \sin \phi=-\sqrt{2 m_{e} T\left(1-\frac{T}{T_{\text {max }}}\right)}\left|\vec{\xi}_{\perp}\right| \sin \phi
\end{gathered}
$$

where $T_{\max }=2 \omega^{2} /\left(m_{e}+2 \omega\right)$ is the maximum electron recoil energy.

\subsection{Active-sterile case}

In this case the three terms of the differential cross section in eq. (13) are different with respect to the active-active case, since sterile neutrinos do not have weak interactions. For instance the purely weak term will consist only of the electron neutrino contribution in eq. (14), while the purely electro-magnetic term in the presence of Dirac-type dipole moments is the well known result

$$
\left(\frac{d \sigma}{d T d \phi}\right)_{e m}=\frac{\alpha^{2}}{2 m_{e}^{2}}\left(\frac{1}{T}-\frac{1}{\omega}\right) \frac{\mu_{e}^{2}+d_{e}^{2}}{\mu_{B}^{2}}
$$

Finally the interference term in the presence of active-sterile dipole moments is

$$
\begin{array}{r}
\left(\frac{d \sigma}{d T d \phi}\right)_{i n t}=-\frac{\alpha G_{F}}{2 \sqrt{2} \pi m_{e} T}\left[\left(\frac{\operatorname{Re}(\mu)+\operatorname{Im}(d)}{\mu_{B}}\right) \vec{p}_{2} \cdot \vec{A}_{S}(T, \omega)\right. \\
\left.+\left(\frac{\operatorname{Re}(d)-\operatorname{Im}(\mu)}{\mu_{B}}\right) \hat{\vec{k}}_{1} \cdot\left(\vec{p}_{2} \times \vec{A}_{S}(T, \omega)\right)\right]
\end{array}
$$

where

$$
\vec{A}_{S}(T, \omega) \equiv\left[g_{e L}+g_{R}\left(1-\frac{T}{\omega}\right)\right] \vec{\xi}_{\perp}^{e \bar{s}}
$$

In the limit when the active-sterile pair form a Dirac neutrino eq. (24) reduces to

$$
\left(\frac{d \sigma}{d T d \phi}\right)_{i n t}=-\frac{\alpha G_{F}}{2 \sqrt{2} \pi m_{e} T}\left[\left(\frac{\mu_{e}}{\mu_{B}}\right) \vec{p}_{2} \cdot \vec{A}_{D}(T, \omega)+\left(\frac{d_{e}}{\mu_{B}}\right) \hat{\vec{k}}_{1} \cdot\left(\vec{p}_{2} \times \vec{A}_{D}(T, \omega)\right)\right]
$$

where

$$
\vec{A}_{D}(T, \omega) \equiv\left[g_{e L}+g_{R}\left(1-\frac{T}{\omega}\right)\right] \vec{\xi}_{\perp}^{e}
$$

The first term in this result was obtained in ref. [13], while the second CP-violating term was given in ref. 22] (see their eq. 9c). 


\section{Test of CP conservation at Hellaz}

We propose to measure solar neutrino-electron scattering in upcoming experiments that will be capable of measuring directionality of the outgoing $e^{-}$(like Hellaz). The relevant observable is the azimuthal distribution of events, namely

$$
\frac{d N}{d \phi}=N_{e} \sum_{i} \Phi_{0 i} \int_{T_{T h}}^{T_{\max }} d T \int_{\omega_{\min }(T)}^{\omega_{\max }} d \omega \lambda_{i}(\omega) \epsilon(\omega) \frac{d \sigma}{d T d \phi}(\omega, T)
$$

where $d \sigma / d T d \phi$ is the complete differential cross section of eq. (13), $\epsilon(\omega)$ is the efficiency of the detector and $N_{e}$ is the number of electrons in the fiducial volume of the detector. The sum in the above equation is done over the solar neutrino spectrum, where $i$ corresponds to the different reactions $i=p p,{ }^{7} \mathrm{Be}, p e p,{ }^{8} \mathrm{~B} \ldots$, characterized by a differential spectrum $\lambda_{i}(\omega)$ and an integral flux $\Phi_{0 i}$.

In the previous section we found the expressions for the differential cross section. The azimuthal distribution of the number of events can be written in a general form as

$$
\frac{d N}{d \phi}=n_{\text {weak }}+n_{\text {em }}+n_{\text {int }} \cos (\phi+\delta)
$$

where $n_{\text {weak }}\left(n_{\text {em }}\right)$ accounts for the weak (electro-magnetic) contributions, while $n_{\text {int }}$ is the interference term. The dependence of the last term on the azimuthal angle $\phi$ is parametrized with $\delta$. Thus a pure $\cos \phi(-\sin \phi)$ dependence corresponds to $\delta=0$ $(\delta=\pi / 2)$. We can define the differential azimuthal asymmetry as

$$
\left.\frac{d A}{d \phi}\right|_{\phi^{\prime}}=\frac{\left.\frac{d N}{d \phi}\right|_{\phi^{\prime}}-\left.\frac{d N}{d \phi}\right|_{\phi^{\prime}+\pi}}{\left.\frac{d N}{d \phi}\right|_{\phi^{\prime}}+\left.\frac{d N}{d \phi}\right|_{\phi^{\prime}+\pi}}=\frac{n_{\text {int }}}{n_{\text {weak }}+n_{e m}} \cos \left(\phi^{\prime}+\delta\right)
$$

where $\phi\left(\phi^{\prime}\right)$ is measured with respect to the direction of the magnetic field $\vec{B}_{\odot}$, which we will assume to be along the positive $x$-axis (see fig. 11). By integrating over $\phi$ one can also define an asymmetry $\mathcal{A}$ as

$$
\mathcal{A}\left(\phi^{\prime}\right)=\frac{\int_{\phi^{\prime}}^{\phi^{\prime}+\pi} \frac{d N}{d \phi} d \phi-\int_{\phi^{\prime}+\pi}^{\phi^{\prime}+2 \pi} \frac{d N}{d \phi} d \phi}{\int_{\phi^{\prime}}^{\phi^{\prime}+\pi} \frac{d N}{d \phi} d \phi+\int_{\phi^{\prime}+\pi}^{\phi^{\prime}+2 \pi} \frac{d N}{d \phi} d \phi}=-A \sin \left(\phi^{\prime}+\delta\right)
$$

where $A \equiv 2 n_{\text {int }} / \pi\left(n_{\text {weak }}+n_{\text {em }}\right)$ is the maximum integrated asymmetry measurable by the experiment, which is manifestly positive. In our previous paper [17] we calculated the 
expected values of $A$ for $p p$ solar neutrinos at Hellaz, for different choices of the survival probability of $\nu_{e}$ 's, in the CP-conserving case of eq. (9).

Let now discuss how the measurement of the azimuthal asymmetry could be carried

out considering that $\vec{B}_{\odot}$ is constant over a given period of time and its direction is known. One should collect events in every $\phi$-bin, where $\phi$ is defined with respect to the positive $x$-axis and then take for different $\phi^{\prime} s$ the ratio $\mathcal{A}(\phi)$ which should show a $\sin \phi$ dependence with a maximum equal to $A$. This will allow us to identify the value of $\delta$.

Note that if we were able to find from the measurements that $\delta \neq 0$ beyond experimental uncertainties, then this would lead to the conclusion that $C P$ is not conserved in the electro-magnetic interactions of neutrinos if we consider Dirac diagonal or Majorana transition dipole moments. In the Dirac transition case the CP phases $\eta_{i}$ of the neutrinos can be chosen so as to have CP conservation for any value of $\delta$. However, Dirac dipole moments do not seem to be favored by theoretical models nor by the existent astrophysical and cosmological constraints [12].

\section{Discussion}

We have shown that the scattering of solar neutrinos on electrons may reveal their CP properties, which are particularly sensitive to their Majorana nature, due to the interference of electro-magnetic and weak amplitudes. We showed how future solar neutrino experiments with good angular resolution and low energy threshold can be sensitive to the resulting azimuthal asymmetries in event number, and could therefore provide valuable information on the CP properties and the nature of the neutrinos, provided the solar magnetic field direction is fixed. Hellaz will be the first experiment which is potentially sensitive to azimuthal asymmetries since the directionality of the outgoing $e^{-}$can be measured. The angular resolution is expected to be $\Delta \theta \sim \Delta \phi \sim 30 \mathrm{mrad} \sim 2^{\circ}$, substantially better than that of Super-Kamiokande. Notice also that the width of the Cerenkov cone defined by the angle $\theta$ is very narrow for high-energy boron neutrinos, as one can see from eq. (22). In contrast, for $p p$ neutrino energies accessible at Hellaz $\left(T_{\max } \simeq 0.26 \mathrm{MeV}\right.$, $T_{t h} \simeq 0.1 \mathrm{MeV}$ ) we estimate that $\theta$ can be as large as $48^{\circ}$. It is important to emphasize here that, while the existence of an asymmetry in event number can be ascribed to a nonzero neutrino electro-magnetic dipole moment, one can not infer any information on the specific issue of CP conservation in the neutrino sector and the nature of neutrinos with- 
out an accurate knowledge of the direction of the solar magnetic field. Such a knowledge is indeed possible except at minimal solar activity periods, when the toroidal magnetic field vanishes.

\section{Acknowledgements}

The authors thank Thomas Ypsilantis for fruitful discussions on the Hellaz experiment. This work has been supported by DGICYT under Grants PB95-1077, by the TMR network grant ERBFMRXCT960090 and by INTAS grant 96-0659 of the European Union. V. S. acknowledges also the support of Generalitat Valenciana and RFBR grant 97-0216501.

\section{References}

[1] J. Schechter and J.W.F. Valle, Phys. Rev. D22 (1980) 2227

[2] J. Schechter and J.W.F. Valle, Phys. Rev. D25 (1982) 2951

[3] L. Baudis et al., Phys. Rev. Lett. 83 (1999) 41

[4] J. Schechter and J.W.F. Valle, Phys. Rev. D23 (1981) 1666

[5] J. Schechter and J.W.F. Valle, Phys. Rev. D24 (1981) 1883; err. ibid. D25 (1982) 283; L. Wolfenstein, Phys. Lett. 107B (1981) 77

[6] R. Shrock, Nucl. Phys. B206 (1982) 359; B. Kayser, Phys. Rev. D26 (1982) 1662

[7] J.F. Nieves, Phys. Rev. D26 (1982) 3152

[8] For reviews and discussion on various models see, for instance R.N. Mohapatra and P.B. Pal, Massive Neutrinos in Physics and Astrophysics (Ed. World Scientific, 1991) and J.W.F. Valle, Gauge Theories and the Physics of Neutrino Mass, Prog. Part. Nucl. Phys. 26 (1991) 91

[9] C.W. Kim and A. Pevsner, Neutrinos in Physics and Astrophysics (Ed. Harwood Academic Publishers, 1993) 
[10] C.S. Lim and W. Marciano, Phys. Rev. D37 (1988) 1368;

E. Kh. Akhmedov, Phys. Lett. B213 (1988) 64

[11] E.Kh. Akhmedov, The neutrino magnetic moment and time variations of the solar neutrino flux, invited talk given at the 4th International Solar Neutrino Conference, Heidelberg, Germany, April 1997, preprint IC/97/49, hep-ph/9705451

[12] G. Raffelt, Stars as laboratories for fundamental physics, (Univ. of Chicago Press, Chicago-London 1996)

[13] R. Barbieri and G. Fiorentini, Nucl. Phys. B304 (1988) 909

[14] See, e.g. W. Grimus and P. Stockinger, Phys. Rev. D57 (1998) 1762

[15] F. Arzarello et al., Preprint CERN-LAA/94-19, College de France LPC/94-28 (1994); J. Seguinot et al., Preprint LPC 95 08, College De France, Laboratoire de Physique Corpusculaire (1995)

[16] M.B. Voloshin and M.I. Vysotsky, Sov. J. Nucl. Phys. 44 (1986) 845;

M.B. Voloshin, M.I. Vysotsky, and L.B. Okun, Sov. Phys. JETP 64 (1986) 446

[17] S. Pastor, J. Segura, V.B. Semikoz and J.W.F. Valle, Phys. Rev. D59 (1999) 013004

[18] F. Mandl and G. Shaw, Quantum Field Theory (Ed. John Wiley and Sons, 1984)

[19] G. Raffelt, Phys. Rev. D39 (1989) 2066

[20] S.M. Bilenky, Introduction to Feynman Diagramas and Electroweak Interactions Physics (Ed. Frontières, 1994)

[21] V.B. Semikoz, Nucl. Phys. B498 (1997) 39

[22] P. Stockinger and W. Grimus, Phys. Lett. B327 (1994) 327 